\title{
Investigation of Neuroprotective Potential of Anti-Diabetic Agents in Ischemic Brain Proteomics Through In-Silico Molecular Simulation Studies
}

\author{
Vishal Chavda 1(D), Vimal Patel ${ }^{2}$ (D), Snehal Patel 1,*(i) \\ 1 Department of Pharmacology, Nirma University, Ahmedabad, Gujarat, India.;chavdavishal2@ gmail.com (V.C.); \\ 2 Department of Pharmaceutics, Nirma University, Ahmedabad, Gujarat, India.;email2vimal.patel@gmail.com (V.P.); \\ * Correspondence: snehalpharma53@gmail.com (S.P.);
}

Scopus Author ID:35278810600

Received: 8.07.2021; Revised: 28.08.2021 Accepted: 2.09.2021; Published: 18.10.2021

\begin{abstract}
Defective neurotransmission, impaired synaptic plasticity, and progressive neurodegeneration triggered by diabetes and stroke. The computational designing of a therapeutic agent, molecular dynamic (MD) simulation, and molecular docking are essential, along with streamlined and inexpensive techniques which enable the potential therapeutic targets for the novel drug discovery process in specific pathology. Molecular docking software such as AutoDock tool v1.5.6, PyRx v8.0, Discovery Studio Visualizer v19.1.0.18287, and PyMOL v2.3.1 has been used for virtual screening and identification of structural based molecular interactions. The 3D co-crystal structures of receptor/target therapeutic proteins (BCl-2, Caspase-3, Caspase-8, Caspase-9, IL6, MAPKERK, PI3K, TGF- $\beta$, TNF$\alpha$, and ZO-1) with attached ligand were gained from the RCSB-PDB website in PDB format. The obtained structures comprise a ligand and a water molecule, which must be removed before docking from the receptor using Discovery Studio Visualizer and finally transformed into macromolecules by AutoDock Vina of PyRx. The active residues were recognized through previously published literature. In-Silico molecular simulation study of anti-diabetic agents with stroke and neurodegenerationassociated proteins gave a strong hypothesis to the neuroprotective actions of the anti-diabetic drugs. Moreover, higher binding energy with all biomarker proteins directs us of exerting strong therapeutic action of the selected class of anti-diabetic agents, i.e., voglibose, saxagliptin, repaglinide, and Dapagliflozin with BCl-2, Caspase-3, Caspase-8, Caspase-9, IL6, MAPK/ERK, PI3K, TGF- $\beta$, TNF- $\alpha$ and ZO-1. The current study elucidates the strong anti-stroke potential of selected anti-diabetic agents and can claim a strong candidature as anti-stroke therapeutics in specific hyperglycemic conditions.
\end{abstract}

Keywords: Anti-diabetic agents; drug discovery; hyperglycemia; ischemia; MD simulation.

(C) 2021 by the authors. This article is an open-access article distributed under the terms and conditions of the Creative Commons Attribution (CC BY) license (https://creativecommons.org/licenses/by/4.0/).

\section{Introduction}

The basic requirement of new drug discovery and development is the structural activity relationship (SAR) and molecular interactions between the receptor and ligand. For computational designing of a therapeutic agent, molecular dynamic (MD) simulation and molecular docking are the most important streamlined and inexpensive techniques. Advances in structural biology techniques such as XRD, NMR, and electrophoretic MD simulation have generated numerous structural bio-molecules and therapeutic agents [1]. The receptor-ligand interface needs to be evaluated at their atomic level fluctuation to understand the SAR and inter-molecular interaction patterns, which could be used to understand the biomolecular functions and development of novel therapeutic agents [2]. Throughout the simulation, each 
particle of receptor and ligand interact with each other for a specific period of time and produces a paragon of the interaction patterns that can be further analyzed for the dynamic nature of the system [3]. MD simulation and molecular docking generated the 3-D structure of the receptor-ligand complex, identifying the active structural conformations, binding affinities/energies, strength and type of interactions (hydrophilic, hydrophobic, electrostatic, van der Waals, etc.), active residual (amino acids) binding sites which reveal mechanism and stability of the complex through how the ligand fit or interact inside the binding pocket of the receptor. The great combination of the experimental model with in-silico methods have expansively been utilized for identification and assessment of biological events, disease condition, physiological process even therapeutic treatment at the cellular and molecular levels [4].

Diabetes and stroke as pre-morbidities are significantly linked with cognitive demur, neurodegenerative and psychological disorders [5,6]. Defective neurotransmission, impaired synaptic plasticity, and progressive neurodegeneration triggered by diabetes and stroke unequivocally increase the odds of cognitive impairment in diabetic victims $[7,8]$. Several cerebrovascular risk factors comprising hyperglycemia, dyslipidemia, hypertension, and genetic, demographic, and lifestyle aspects contribute to varying degrees to make up the twofold high chance for ischemic stroke in diabetic patients compared to non-diabetic individuals [9]. The four main direct reasons for diabetic patients to acquire stroke are oxidative stress, endothelial dysfunction, systemic inflammation, and arterial stiffness [10]. The anti-diabetics that lower hyperglycemia through different mechanisms is glucagon-like peptide-1 (GLP-1) analogs, insulin sensitizer (i.e., rosiglitazone), and glucose synthesis inhibitor (i.e., metformin), also show favorable effects on neuroplasticity and behavior [11]. It has been reported that the use of anti-diabetic agents like sulfonylurea by acute ischemic stroke patients with coexisting diabetes is less likely to have symptomatic hemorrhagic transformation[12]. Anti-diabetic drugs such as voglibose, saxagliptin, repaglinide, and Dapagliflozin have shown prominent action in hyperglycemic conditions. Stroke activates various neurodegenerative mechanisms through inflammation and apoptotic changes and up or down-regulate a few protein expressions, which play a vital role in stroke pathogenesis. Diabetic individuals have already altered mechanisms that interplay in stroke pathogenesis. In the current study, we aimed to investigate the role of anti-diabetic agents in altered stroke proteomics through MD simulation studies.

\section{Materials and Methods}

\subsection{Preparation of the receptor/target and ligand for the docking.}

The mechanism of interactions of a ligand with receptor/target therapeutic protein was done by in-silico molecular docking programs (steps including preparation of protein binding site and ligand, docking process, structural-based virtual screening, and molecular dynamic simulation was carried out). The accuracy of these docking mechanisms remains intricate due to an incomplete understanding of the ligand-binding process [13]. Molecular docking software, such as AutoDock tool v1.5.6, PyRx v8.0, Discovery Studio Visualizer v19.1.0.18287, and PyMOL v2.3.1, has been used for virtual screening and identification of structural based molecular interactions. The 3D co-crystal structures of receptor/target therapeutic proteins (BCl-2, Caspase-3, Caspase-8, Caspase-9, IL6, MAPKERK, PI3K, TGF$\beta$, TNF- $\alpha$ and ZO-1) with attached ligand were gained from the RCSB-PDB website 
(http://www.rcsb.org/) in PDB format. The obtained structures comprise ligand and water molecules, which must be removed before docking from the receptor using Discovery Studio Visualizer and finally transformed into macromolecules by AutoDock Vina of PyRx. The active site residues of all described receptors were determined by a literature review [14]. The list of active binding site residues (amino acid) of mentioned proteins is mentioned in Table 1. The 3D structure of all the drug molecules (Voglibose, Saxagliptin, Repaglinide, and Dapagliflozin) in SDF format were obtained from the PubChem (https://pubchem.ncbi.nlm.nih.gov/) database. Then molecules are minimized and converted into PDBQT file using AutoDock Vina of PyRx [15].

Table 1. Summary of the active binding site (residual amino acid) of listed protein molecules

\begin{tabular}{l|l|l|l|l|l|l|l|l} 
Proteins & Active site residues (amino acid) \\
\hline BCL2 & Tyr9 & Asp10 & Asn11 & Asn182 & His186 & Gly194 & Trp195 & \\
\hline Caspase3 & Ser65 & Ser205 & Trp206 & Arg207 & Asn208 & Ser209 & & \\
\hline Caspase8 & --- & & & & \\
\hline Caspase9 & Arg75 & Asn78 & Va185 & Arg86 & Asn87 & Lys88 & Asn89 & \\
\hline IL6 & Leu163 & Gly164 & Thr165 & Ser168 & Arg180 & Trp181 & His200 & \\
\hline MAPKERK & Gln105 & Asp106 & Thr110 & Asp111 & Lys114 & Ser153 & Asn154 & Asp167 \\
\hline TNF-A & Leu57 & Tyr59 & Ser60 & Tyr119 & Leu120 & Gly121 & Gly122 & \\
\hline TGF-B & Leu49 & Asn53 & Arg56 & & & & & \\
\hline ZO-1 & --- & & \\
\end{tabular}

\subsection{Docking process.}

The drug ligands were docked with the receptors binding site of PDBQT file using AutoDock vina of PyRx software. The first step to prepare PDBQT files of protein and ligands and grid box formation was carried out using the AutoDock MGT tool console. Gasteiger charges and polar hydrogen were assigned by the Autodock4 tool. Grid boxes were prepared by accessing the Autogrid-4 tool, which was proceeded before docking. After the selection of PDBQT files of protein and ligands, docking preference was originated by AutoDock vina wizard. The obtained grid box was adjusted to the selected active site of the protein. Then proceed for the vina simulation for structural interactions. It provided the negative value of ligands binding affinity/efficacy of each orientation with the receptor proteins measured in $\mathrm{kcal} / \mathrm{mol}$, a unit of docking score. It ranked and provided all possible conformation/orientation of receptor-ligand interactions. Amongst binding affinities, each orientation's upper and lower value of root-mean-square deviation (RSMD) has been provided in ascending order [16].

\subsection{Virtual screening and molecular dynamics simulation study.}

Interactions and molecular simulation of docked ligands with the different proteins were completed by PyMol molecular graphics system. The H-bond interactions, hydrophobic interactions, and binding patterns of docked molecules were examined by Discovery Studio Visualizer [13,17].

\section{Results}

All the anti-diabetic drugs (Voglibose, Saxagliptin and Repaglinide, and Dapagliflozin) were assigned to AutoDock vina of PyRx software for docking with different targeted proteins. PyRx software docked the possible different conformation/orientation of the drug ligand with the active site residues of the targeted receptors. Molecular dynamic simulation and validation of all the docked orientation were done by PyMol molecular graphics system and Discovery 
studio visualizers. In final outcomes from all orientation, zero orientations of the docked ligands were shown lowest binding energy with null RMSD value defined the best fitting interaction result amongst all orientation[14]. The interaction binding affinities of listed drugs with designated proteins were summarized in Table 2.

Table 2. Summary of the ligand-protein binding affinities/docking score ( $\mathrm{kcal} / \mathrm{mol})$.

\begin{tabular}{l|l|l|l|l}
\multirow{2}{*}{ Proteins } & \multicolumn{4}{|c}{ Ligand binding affinities (kcal/mol) } \\
\cline { 2 - 5 } & Voglibose & Saxagliptin & Repaglinide & Dapagliflozin \\
\hline Bcl-2 & -5.6 & -7.0 & -6.9 & -7.2 \\
\hline Caspase 3 & -5.6 & -6.6 & -6.3 & -6.6 \\
\hline Caspase 8 & -4.8 & -7.0 & -7.5 & -6.9 \\
\hline Caspase 9 & -3.7 & -4.0 & -3.7 & -4.4 \\
\hline IL 6 & -6.6 & -7.0 & -7.9 & -7.3 \\
\hline MAPK/ERK & -5.8 & -7.4 & -7.8 & -8.2 \\
\hline TNF-Alpha & -5.0 & -7.3 & -5.3 & -3.8 \\
\hline TGF-Beta-1 & -3.4 & -3.3 & 4.8 & -3.7 \\
\hline ZO-1 & -4.5 & -6.1 & -5.7 & -5.8
\end{tabular}

All the mentioned drugs were shown binding efficacies to the listed protein molecules. The binding of each drug ligand with different active site residues of the receptor hasbeen achieved by intermolecular chargesassigned to both during the preparation method. $\mathrm{BCl}-2$ receptor has non-specific residues; still, all four drugs have an affinity toward the protein molecule. The active binding site of $\mathrm{BCl}-2$ was determined by blind docking. For blind docking, the process of grid box formation was done by selecting the whole protein molecule. After the docking process, the vina wizard had been generated the tabular report of the possible docked orientation/conformation of ligand binding interaction along with binding affinity (kcal/mol), upper and lower bound RMSD values of each orientation[15]. The zero orientation with the lowest binding affinity was extracted and fetched with the receptor to study molecular dynamic simulation and virtual analysis using the PyMol and Discovery studio visualizer software program[13]. The interactions and binding affinities of the different anti-diabetic drugs with active site residues of BCl-2 protein are shown in Figure 1 and Table 2. Antidiabetic drugs showed explicit binding interaction with $\mathrm{BCl}-2$ protein. Anti-diabetic drug dapagliflozin shows a good lowest binding affinity $-7.2 \mathrm{kcal} / \mathrm{mol}$ with $\mathrm{BCl}-2$ as compared to another drug. The Dapagliflozin has H-bond interaction within the binding pocket of BCl-2 protein such as Asp10, Asn11, Asn182, and His186 amino acids, andhydrophobic amide bond interactions with Thr7, Tyr9 and Gly193 amino acids [9,10]. The similar binding patterns were observed in voglibose and repaglinide with binding affinities -5.6 and $-6.9 \mathrm{kcal} / \mathrm{mol}[18]$. But the saxagliptin shows random interactions outside the binding pocket $\mathrm{BCl}-2$ with binding affinity $-7.0 \mathrm{kcal} / \mathrm{mol}$. The active site residues of $\mathrm{BCl}-2$ involved in the binding with saxagliptin are Glu13, Ala43, Glu46, Thr47, Ser49 amino acids [19].

The binding affinities of voglibose, saxagliptin, repaglinide, and Dapagliflozin against Caspase- 3 protein were found to be $-5.6,-6.6,-6.6$, and $-6.3 \mathrm{kcal} / \mathrm{mol}$, respectively, as shown in Table 2. The binding pattern of these drugs with active site residues of Caspase-3 protein is shown in Figure 2. The voglibose has an H-bond interaction within the binding pocket of Arg64 and Arg207 amino acids and hydrophobic interaction with Tyr204 and Trp206 amino acids [20]. Dapagliflozin and saxagliptin show similar binding affinities $-6.6 \mathrm{kcal} / \mathrm{mol}$ with Caspase3 protein, but a different pattern with active site residues. The Dapagliflozin has an H-bond interaction within the binding pocket of Arg208, Asn207, and Phe250 amino acids, Hydrophobic Alkyl binding interaction with His121 and Cys163 amino acids. 


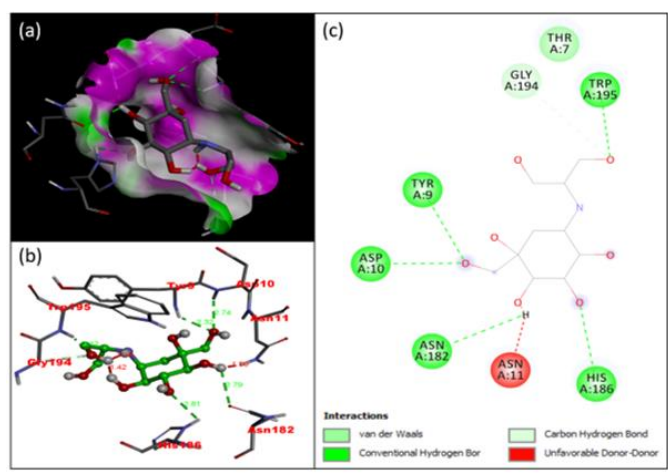

(a) Voglibose

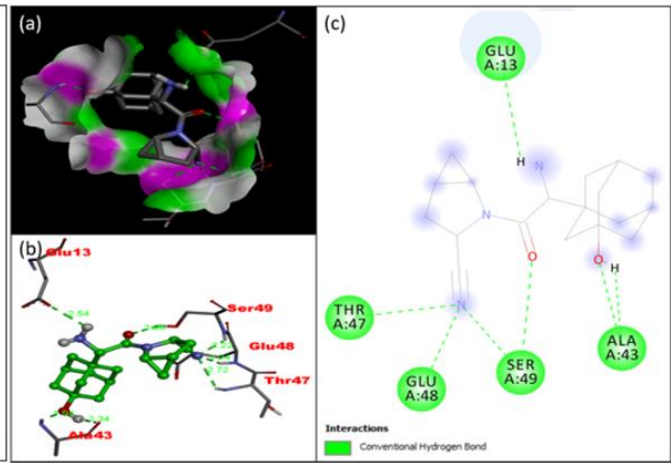

(b) Saxagliptin

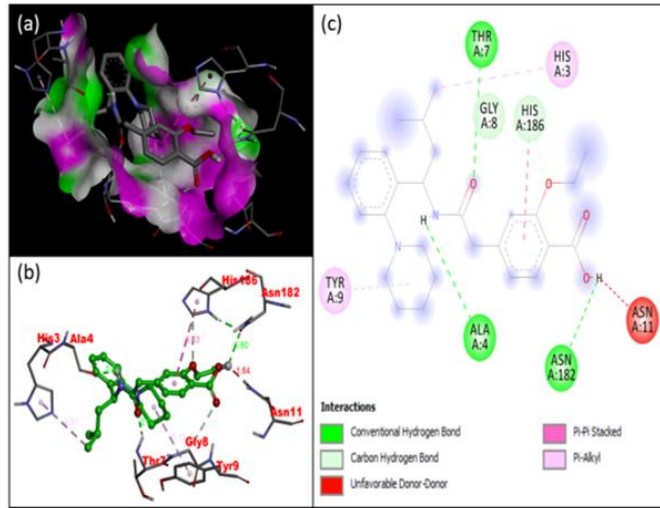

(c) Repaglinide

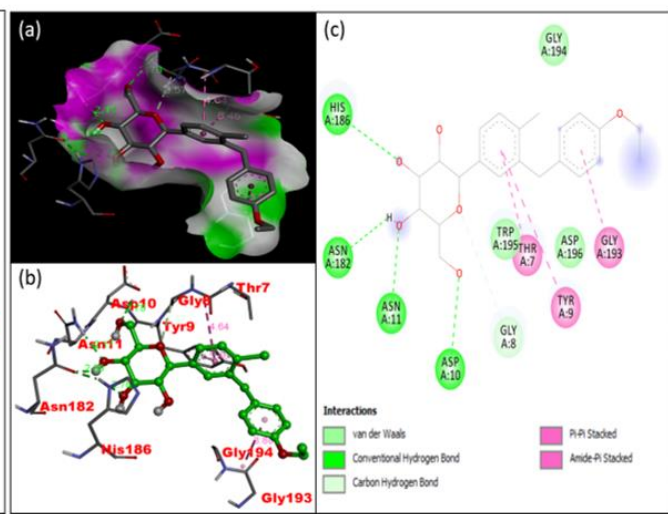

(d) Dapagliflozin

Figure 1. Molecular interaction of anti-diabetic drugs with active site residues of Bcl-2 protein (PDB:1g5m): (a) voglibose; (b) saxagliptin; (c) repaglinide; (d) dapagliflozin.

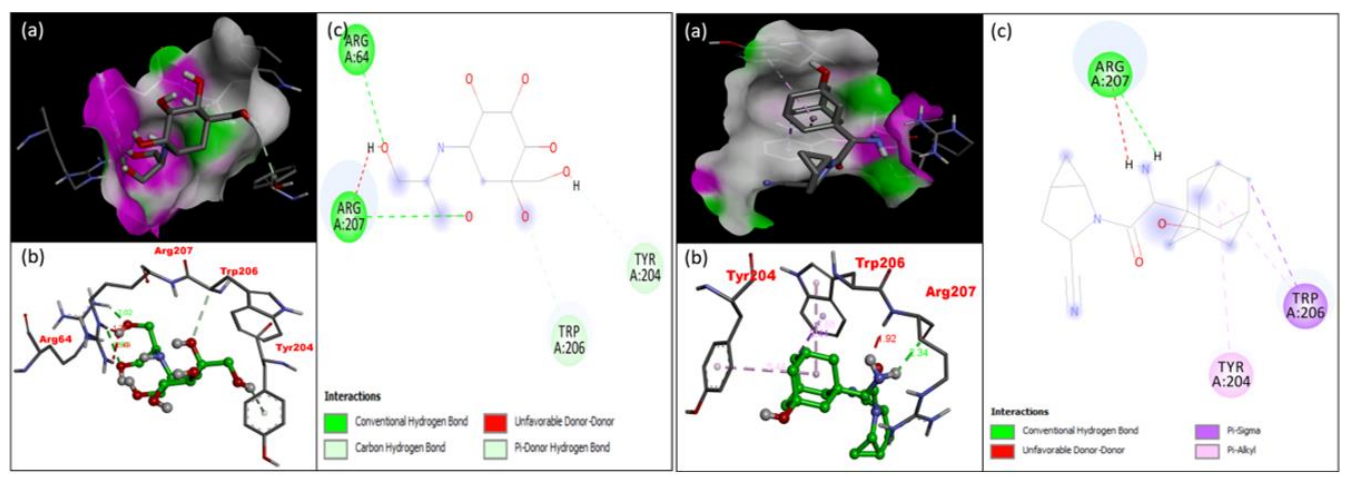

(a) Voglibose

(b) Saxagliptin

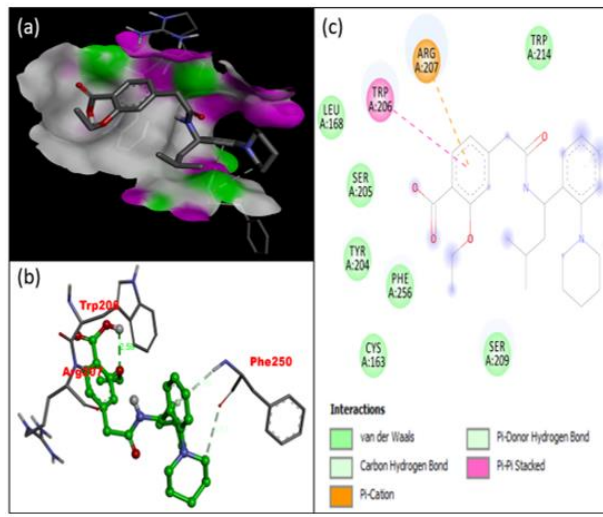

(c) Repaglinide

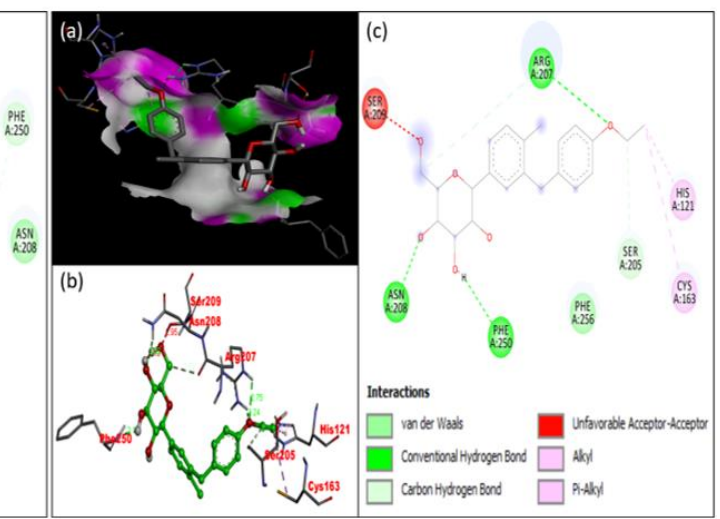

(d) Dapagliflozin

Figure 2. Molecular interaction of anti-diabetic drugs with active site residues of Caspase-3 protein (PDB:4qud): (a) voglibose; (b) saxagliptin; (c) repaglinide; (d) dapagliflozin. 
The saxagliptin has an H-bond interaction with Arg207 amino acid and hydrophobic Pi-alkyl and Pi-sigma interaction with Tyr204 and Trp206 amino acids of Caspase-3 protein. At the same time, Repaglinide has shown binding affinity $-6.3 \mathrm{kcal} / \mathrm{mol}$ with a hydrophobic interaction pattern. It has shown Pi-Pi stacking and Pi-cation interaction with Trp206 and Arg207 amino acids of Caspase-3 protein[21,22].

The active site residues of Caspase- 8 protein were not found in the protein drug bank database. However, we perform blind docking of each drug molecule with Caspase- 8 protein. The binding affinities of voglibose, saxagliptin, repaglinide, and Dapagliflozin were found to be $-4.8,-6.9,-7.0$, and $-7.5 \mathrm{kcal} / \mathrm{mol}$ against Caspase- 8 protein, respectively, through blind docking are shown in Table 2. The binding interactions of these drugs with different residues through blind docking with Caspase- 8 are shown in Figure 3. The voglibose has H-bond interaction within the binding pocket of Asp15, Asp18, Ile89, Tyr106, and Glu110 amino acids. Dapagliflozin only shows binding hydrophobic Alkyl binding interactions with Lys 23 and Leu27 amino acids and Pi-Pi stacking interaction with Phe24 amino acid. Similarly, Repaglinide shows hydrophobic Alkyl binding interactions with Leu150, Ile108, Val112, and Leu117 amino acids and one Pi-sigma interaction with Phe120 amino acid of Caspase-8 protein. The saxagliptin has shown H-bond interaction with Ser109 amino acid and hydrophobic Alkyl binding interaction with Phe24, Leu105, Val112, and Phe120 amino acids [23].

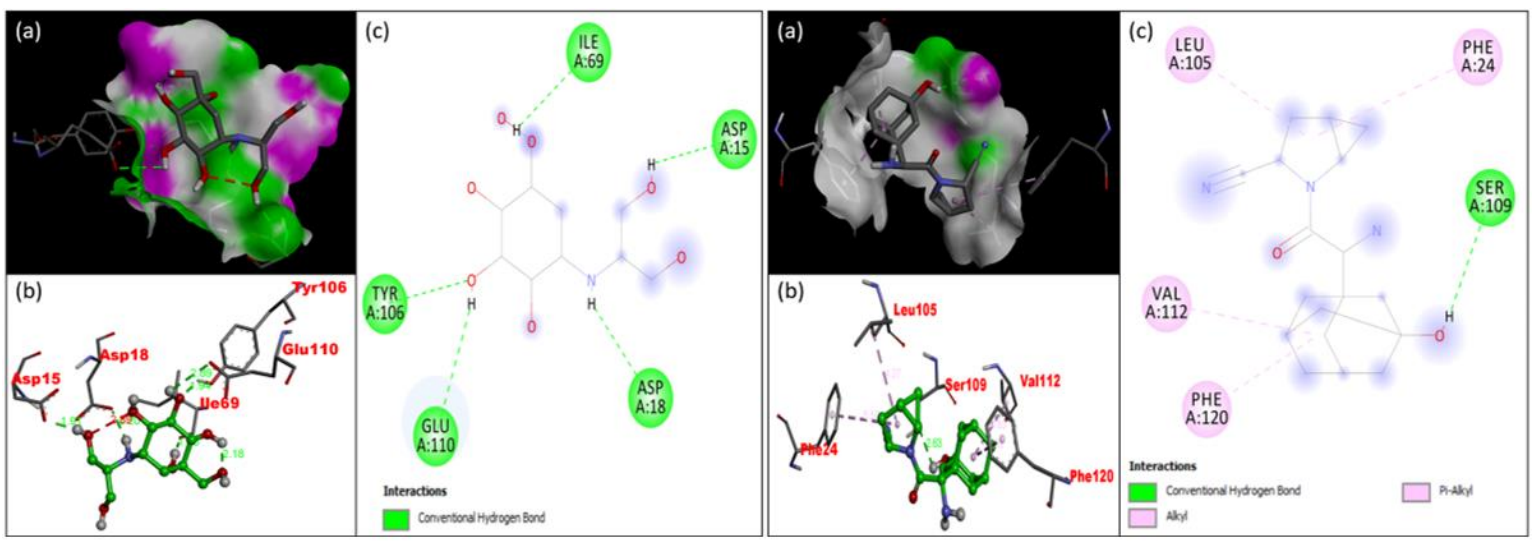

(a) Voglibose

(b) Saxagliptin

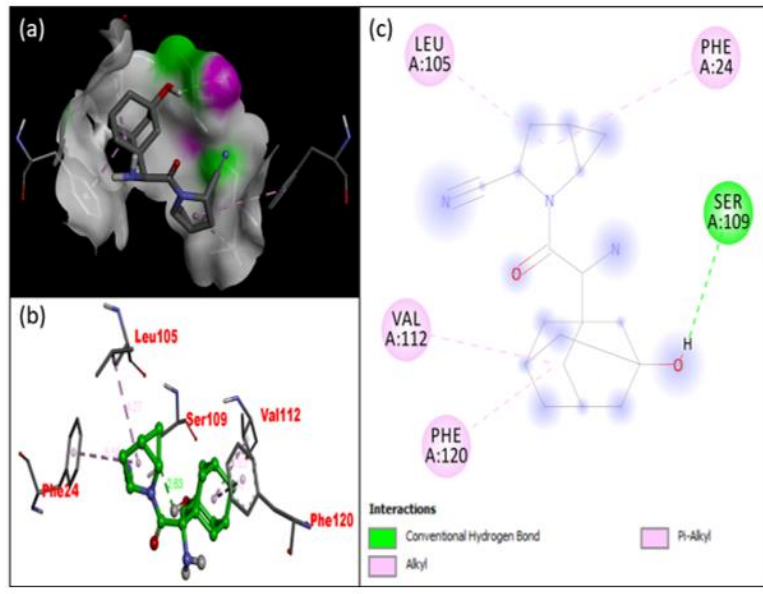

(c) Repaglinide

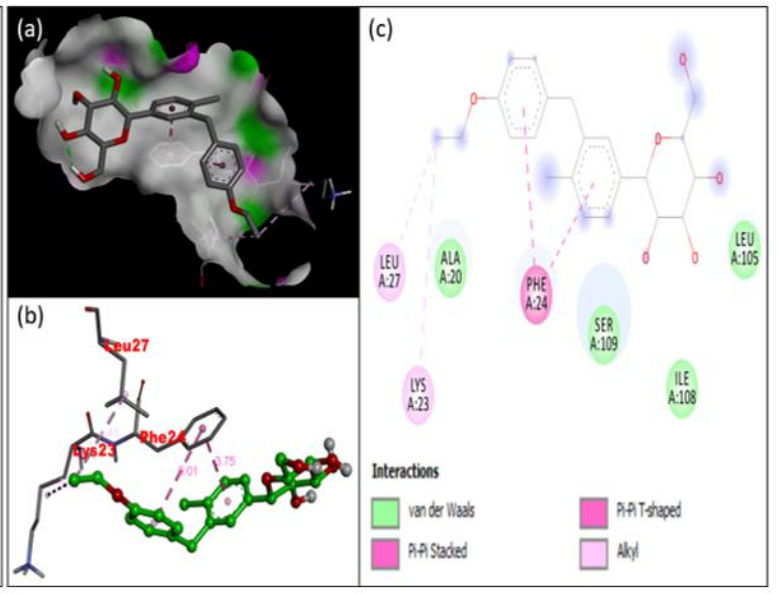

(d) Dapagliflozin

Figure 3. Molecular interaction of anti-diabetic drugs with active site residues of Caspase-8 protein (PDB:6agw): (a) voglibose; (b) saxagliptin; (c) repaglinide; (d) dapagliflozin. 
The binding affinities of voglibose, saxagliptin, Repaglinide, and Dapagliflozin against Caspase-9 protein were found to be $-3.7,-4.4,-4.0$, and $-3.7 \mathrm{kcal} / \mathrm{mol}$, respectively, as shown in Table 2. The binding pattern of these drugs with active site residues of Caspase-9 protein is shown in Figure 4. The voglibose has shown potential H-bond interactions with binding pocket of Caspase-9 protein comprise Val85 and Asn87 amino acids. The Dapagliflozin has shown Hbond interactions within the binding pocket of Val85, Asn87, and Lys88 amino acids and hydrophobic Alkyl interactions with Arg75, Arg79, and Arg86 amino acids of Caspase-9 protein. Similarly, saxagliptin has shown H-bond interaction within the binding pocket of Arg75, Val85, and Lys88 amino acids. In contrast, Repaglinide has shown three different interactions: H-bond interaction with Glu106 amino acid, hydrophobic Pi-Alkyl interaction with Lys88, and hydrophobic Pi-cation interaction with Arg86 amino acid of Caspase-9 protein[24].

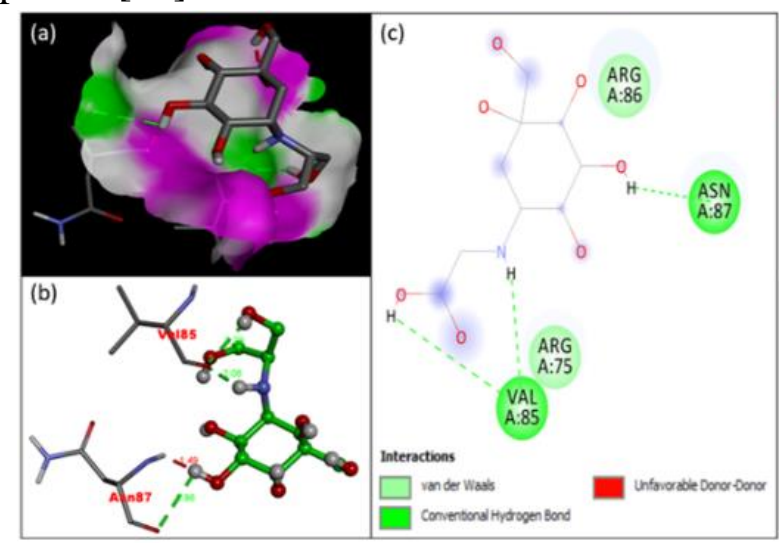

(a) Voglibose

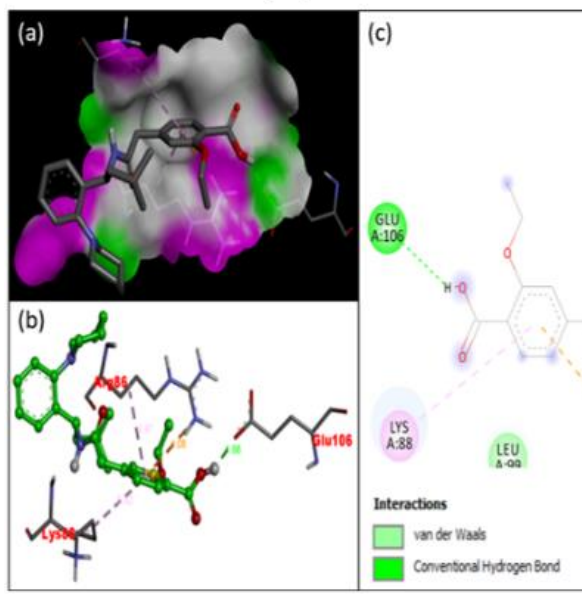

(c) Repaglinide
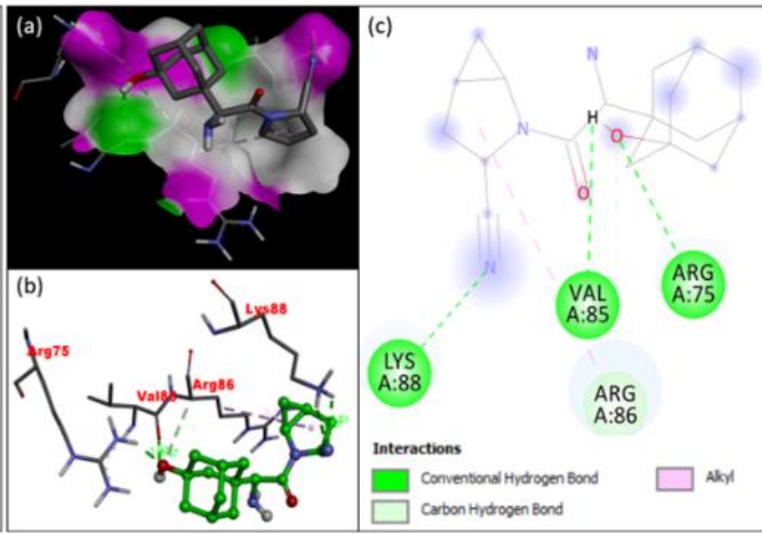

(b) Saxagliptin

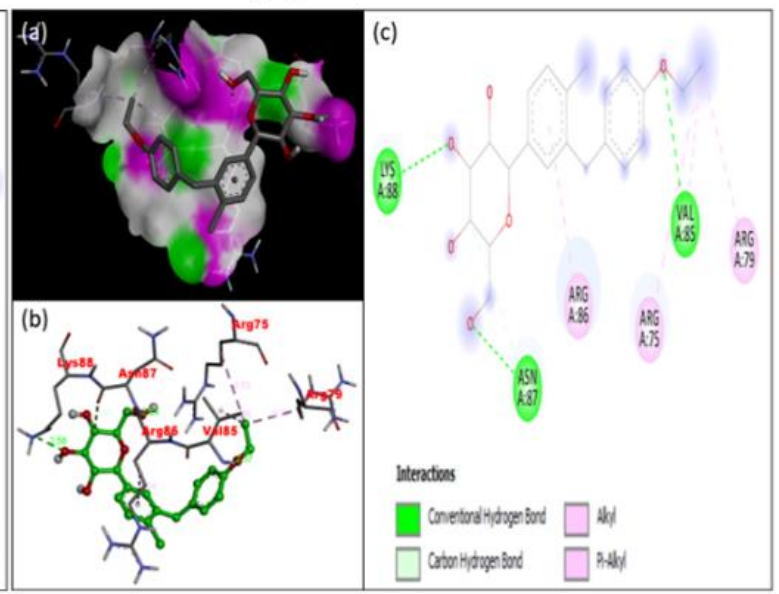

(d) Dapagliflozin

Figure 4.Molecular interaction of anti-diabetic drugs with active site residues of Caspase-9 protein (PDB:5iab): (a) voglibose; (b) saxagliptin; (c) repaglinide; (d) dapagliflozin.

The binding affinities of voglibose, saxagliptin, repaglinide, and Dapagliflozin against Il-6 protein were found to be $-6.6,-7.3,-7.0$, and $-7.9 \mathrm{kcal} / \mathrm{mol}$, respectively, as seen in Table 2. This binding pattern of these drugs with IL-6 protein is shown in Figure 5. Here all the drugs showed binding interactions with non-active residues of IL- 6 proteins. IL- 6 has two different activation signaling pathways; both act on a similar receptor subunit, but their biological responses are diverse. Hence, the effect of IL- 6 on glucose metabolism and insulin sensitivity distinctly varies in the different tissue and situation. The controversial role of IL-6 on insulin resistance and the development of diabetes is still unclear[25]. The voglibose has shown 
potential H-bond interactions with non-binding pocket IL-6 protein includes Arg11, Gly12, Asp13, Phe14, Thr15, Thr35, and Arg188 amino acids. The Dapagliflozin has shown only hydrophobic binding interaction with IL-6 protein includes Pi-sigma interaction with Ile52 amino acid and Pi-alkyl interaction Cys78, Cys88, Leu89, and Ile92 amino acids. The saxagliptin also observed non-pocket H-bond interactions with Leu153 and Lys156 amino acids and exhibited hydrophobic alkyl interactions with Leu152 and Arg165 amino acids. At the same time, Repaglinide has shown three different interaction patterns: H-bond interaction with Ser93 amino acid, hydrophobic Pi-sigma interactions with Ile52, Phe132, and Val136 amino acids, and hydrophobic alkyl interactions with Leu89, Leu90, and Ile142 amino acids of IL-6 protein[26].

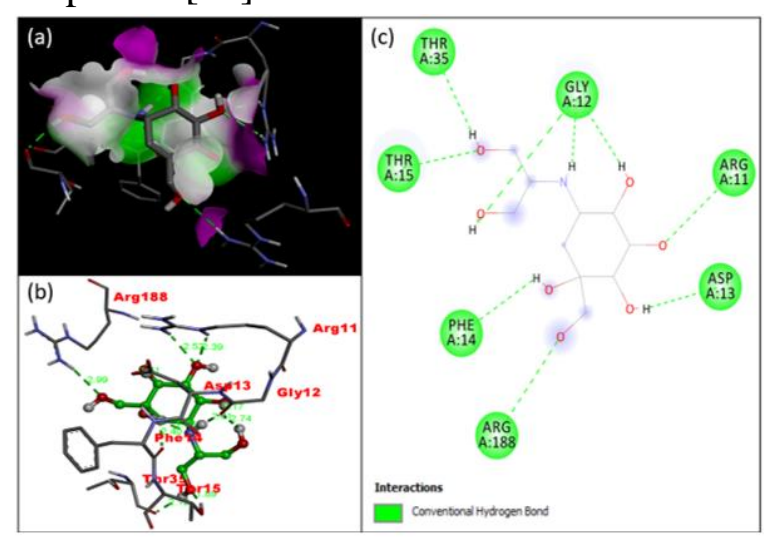

(a) Voglibose

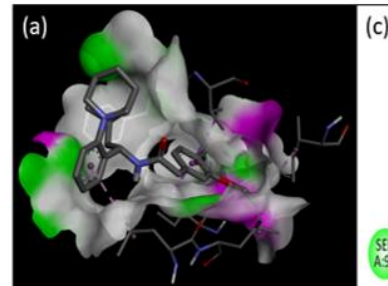

(b)

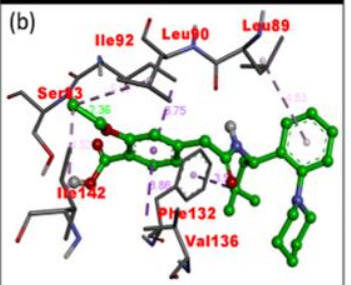

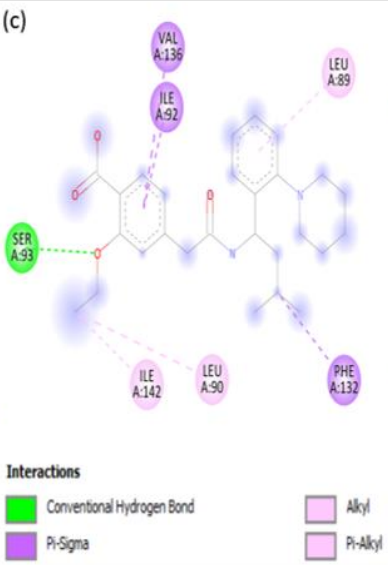

(c) Repaglinide

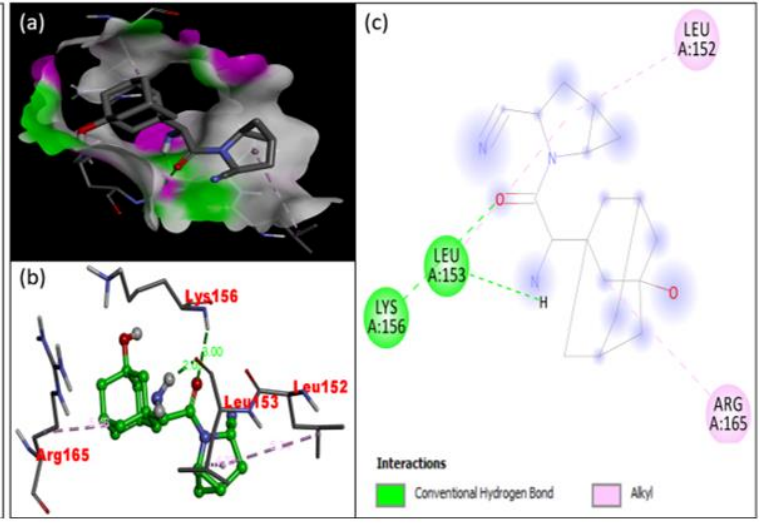

(b) Saxagliptin

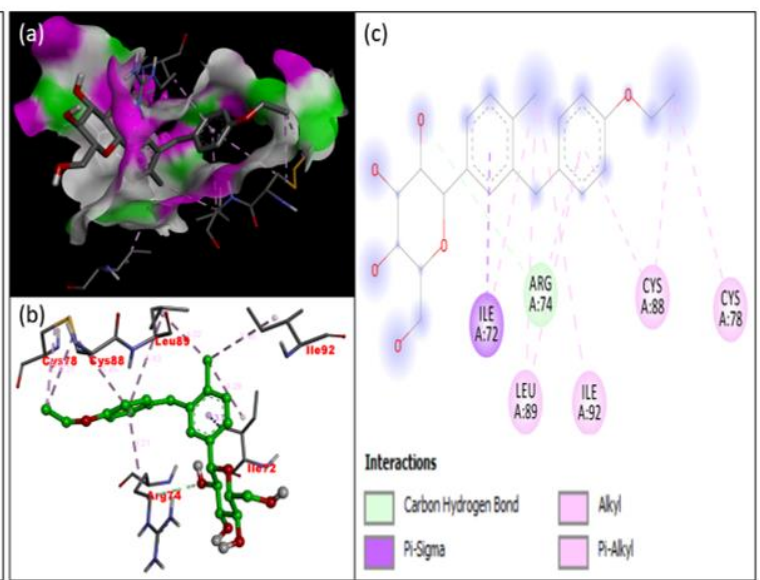

(d) Dapagliflozin

Figure 5. Molecular interaction of anti-diabetic drugs with active site residues of IL-6 protein (PDB:213y): (a) voglibose; (b) saxagliptin; (c) repaglinide; (d) dapagliflozin.

The binding affinities of voglibose, saxagliptin, repaglinide, and Dapagliflozin against MAPKERK protein were found to be $-5.8,-8.2,-7.4$, and $-7.8 \mathrm{kcal} / \mathrm{mol}$, respectively, as shown in Table 2. The binding pattern of these drugs with active site residues of MAPKERK protein is shown in Figure 6. The voglibose has shown potential H-bond interactions with binding pockets of Asp106, Asp111, Lys114, Ser153, and Cys166 amino acids. The Dapagliflozin has shown H-bond interactions with inactive residues amino acids such as Lys54 and Met108 amino acids and hydrophilic alkyl interactions with Tyr36, Val39, and Ile56 amino acids. In comparison, saxagliptin has shown H-bond interaction within binding pocket Ser153 and Asp167 amino acids along with non-active residual sites such as Gly37, Glu71, and hydrophobic alkyl interactions with Val36 and Tyr36 amino acids. The repaglinide has shown different binding interactions outside the binding pocket like $\mathrm{H}$-bond interaction with Met108 
amino acid, hydrophobic Pi-sigma interactions with Leu156 amino acids, and Pi-alkyl interaction with Val39 and Cys166 amino acids of the MAPKERK protein[27].
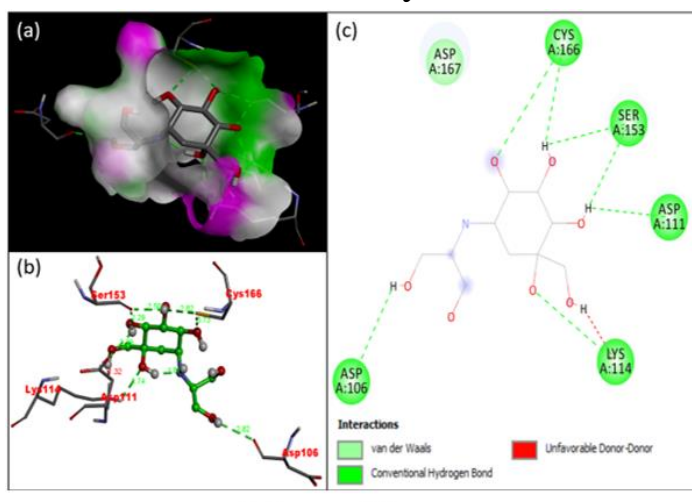

(a) Voglibose
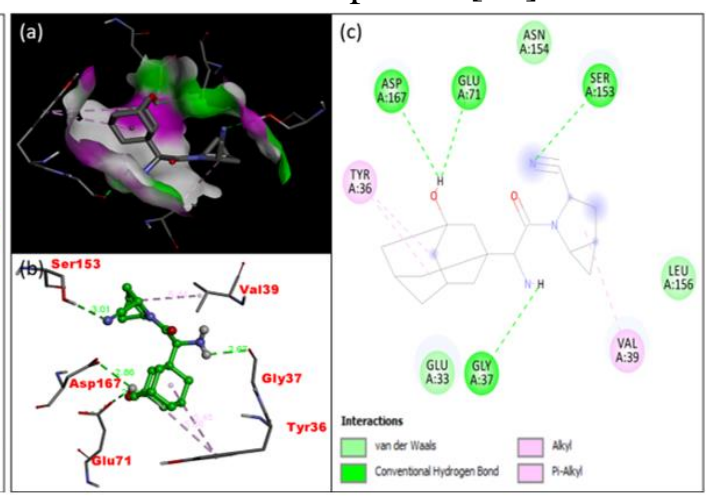

(b) Saxagliptin

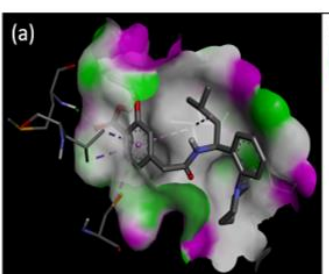

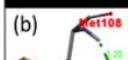

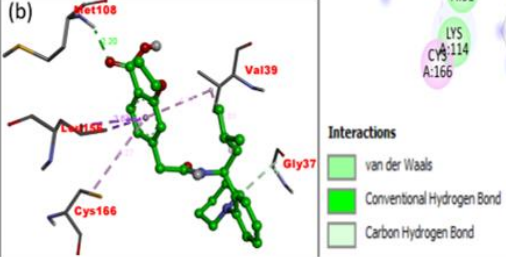

(c) Repaglinide

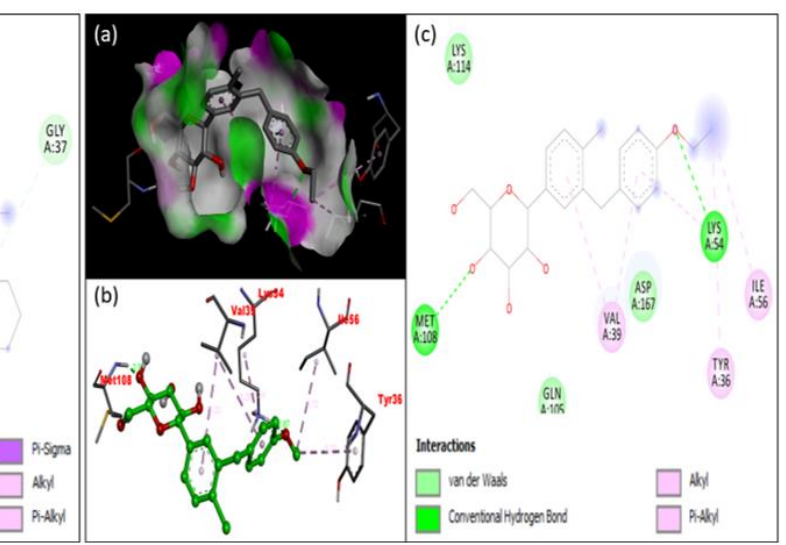

(d) Dapagliflozin

Figure 6.Molecular interaction of anti-diabetic drugs with active site residues of MAPK/ERK protein (PDB:4h3p): (a) voglibose; (b) saxagliptin; (c) repaglinide; (d) dapagliflozin.

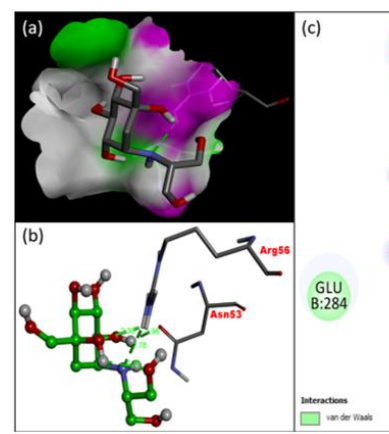

(a) Voglibose

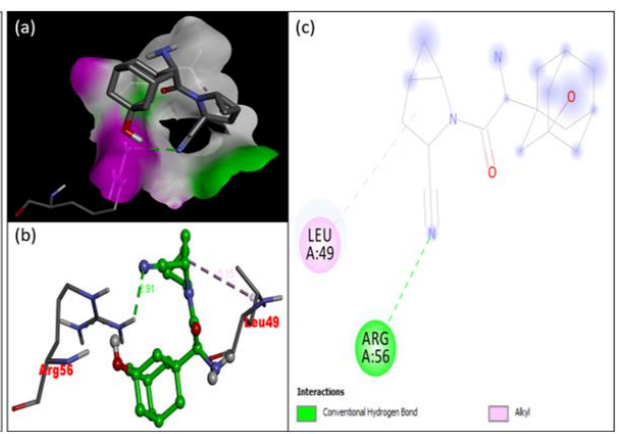

(b) Saxagliptin

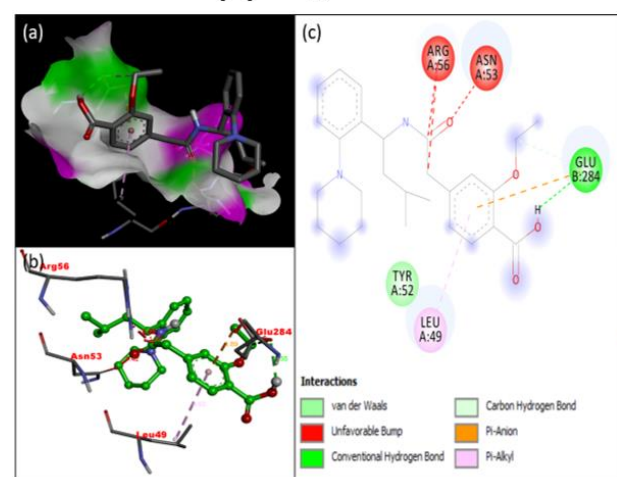

(c) Repaglinide

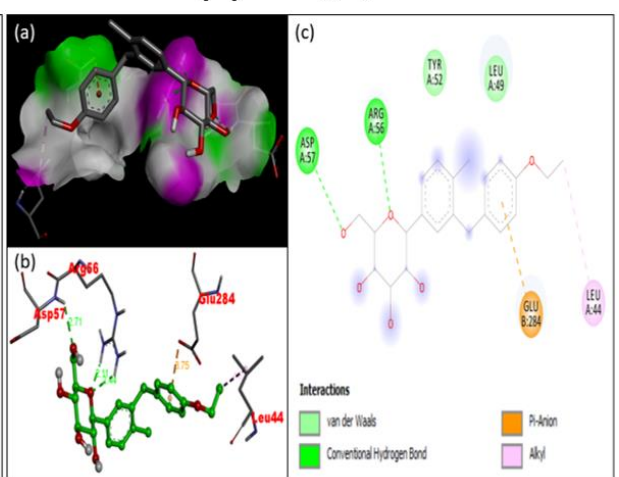

(d) Dapagliflozin

Figure 7. Molecular interaction of anti-diabetic drugs with active site residues of TGF- $\beta$ protein (PDB:3rjr): (a) voglibose; (b) saxagliptin; (c) repaglinide; (d) dapagliflozin. 
The binding affinities of voglibose, saxagliptin, repaglinide, and Dapagliflozin against TGF- $\beta$ protein were found to be $-3.4,-3.7,-3.3$, and $-4.8 \mathrm{kcal} / \mathrm{mol}$, respectively, as shown in Table 2 . The binding pattern of these drugs with active site residues of TGF- $\beta$ protein is shown in Figure 7. The voglibose has shown binding interactions within the binding pocket of Asn53 and Arg56 amino acids. The saxagliptin shows three different types of interactions: H-bond interactions with Arg56 and Asp57 amino acids near the binding pocket, two hydrophobic Pianion and Pi-alkyl interactions with Glu284 and Leu44 amino acids, respectively. Similarly, Dapagliflozin showed two different interactions: H-bond interaction with Arg56 amino acid and hydrophobic alkyl interaction with Leu49 amino acid. At the same time, Repaglinide has shown unfavorable interaction within Asn53 and Arg56 amino acids' binding pocket and hydrophobic Pi-alkyl interaction with Leu49 amino acid. Glu284 amino acid has also exhibited two different interactions, such as H-bond and hydrophobic Pi-anion [28].

The binding affinities of voglibose, saxagliptin, repaglinide, and Dapagliflozin against TNF- $\alpha$ protein were found to be $-5.0,-3.8,-7.3$, and $-5.3 \mathrm{kcal} / \mathrm{mol}$, respectively, shown in Table 2. The binding pattern of these drugs with active site residues of TNF- $\alpha$ protein is shown in Figure 8. The voglibose has shown binding interactions within active site Ser60 and outside Gln61 and Tyr151 amino acids of the TNF- $\alpha$ protein. The Dapagliflozin has two types of hydrophobic interactions, in which Tyr59 amino acid shows both Pi-Pi and Pi-alkyl interactions. While other amino acids Tyr119 and Leu120, have only Pi-Pi interactions, and Tyr151 has only Pi-alkyl interaction. The saxagliptin has shown H-bond interaction within the binding pocket of Ser60 amino acid and hydrophilic Pi-alkyl interactions with Leu57, Tyr59, and Tyr119 amino acids. Similar kinds of interactions also were observed in Repaglinide. The Amino acids Leu57 and Tyr59 have exhibited hydrophobic interactions, such as Pi-sigma and Pi-alkyl[29,30].

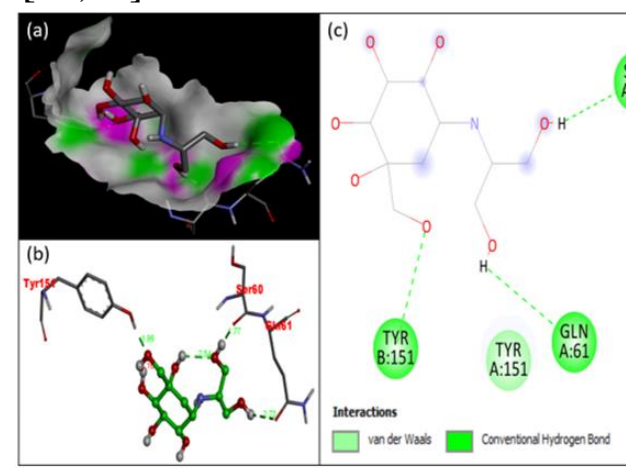

(a) Voglibose

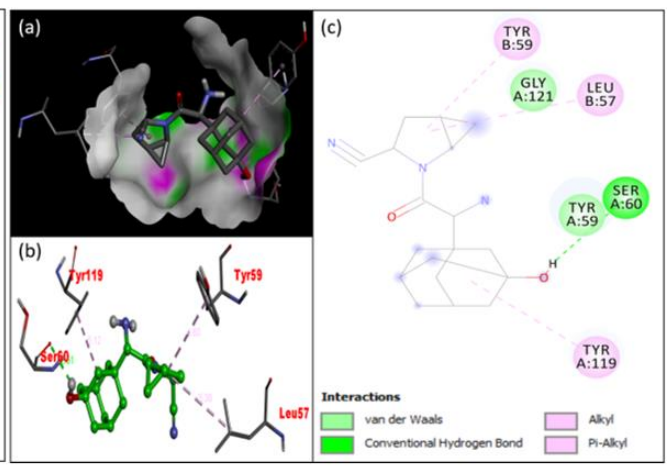

(b) Saxagliptin

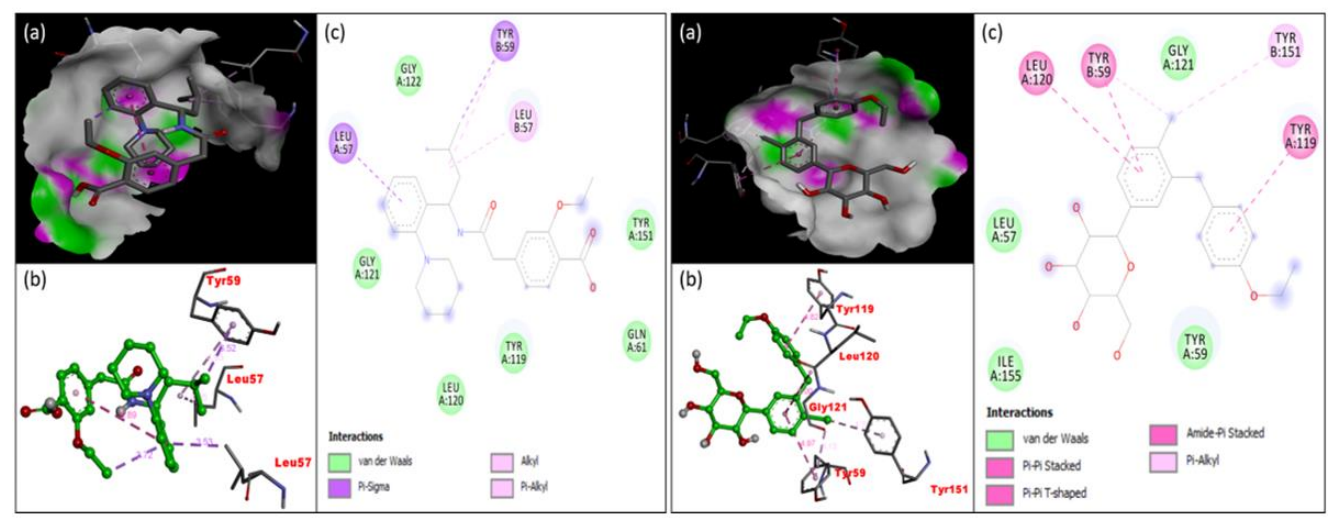

(c) Repaglinide

(d) Dapagliflozin

Figure 8. Molecular interaction of anti-diabetic drugs with active site residues of TNF- $\alpha$ protein (PDB:2az5): (a) voglibose; (b) saxagliptin; (c) repaglinide; (d) dapagliflozin. 
The active site residues of $\mathrm{ZO}-1$ protein were not found in the protein drug bank database. However, we perform blind docking of each drug molecule with $\mathrm{ZO}-1$ protein. The binding affinities of voglibose, saxagliptin, repaglinide, and Dapagliflozin were found to be $4.5,-5.8,-6.1$, and $-5.7 \mathrm{kcal} / \mathrm{mol}$ against $\mathrm{ZO}-1$ protein, respectively through blind docking, as shown in Table 2 . The binding interactions of these drugs with different residues through blind docking with ZO-1 are shown in Figure 9. The voglibose has an H-bond interaction within the binding pocket of Ser6, Met7, and Lys8 amino acids. In contrast, Dapagliflozin has shown three binding interactions: H-bond interaction with Arg13, hydrophobic Alkyl binding interaction with Pro4, and Pi-sigma interaction with Pro40 amino acids. Similarly, repaglinide had shown H-bond interactions with Arg65, hydrophobic Alkyl binding interactions with Arg21 and Leu73, and one Pi-sigma interaction with Val69 amino acids of ZO-1 protein. The saxagliptin showed H-bond interaction with Ser6, Met7, and Lys87 amino acids and hydrophobic Alkyl binding interaction withLys87 amino acid.

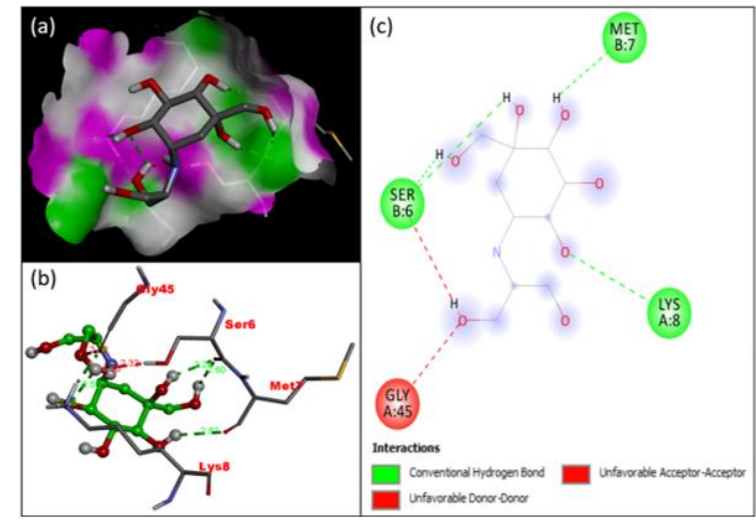

(a) Voglibose

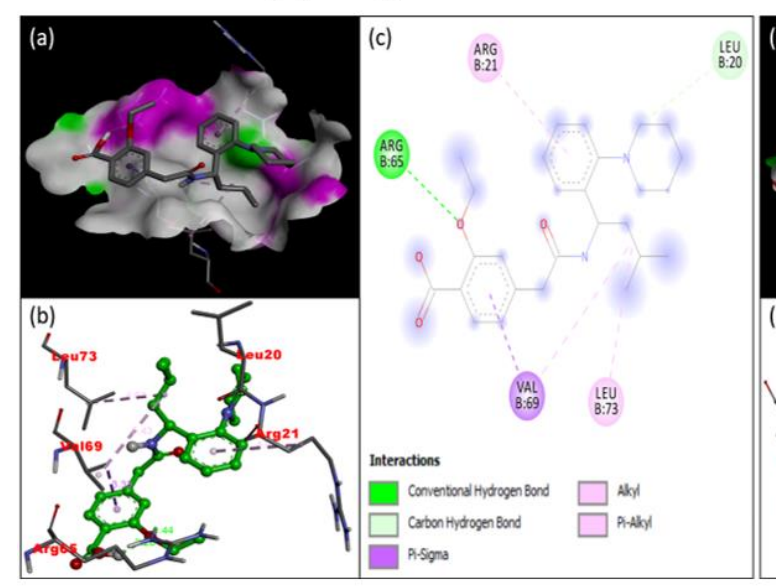

(c) Repaglinide

(c) Repaglinide

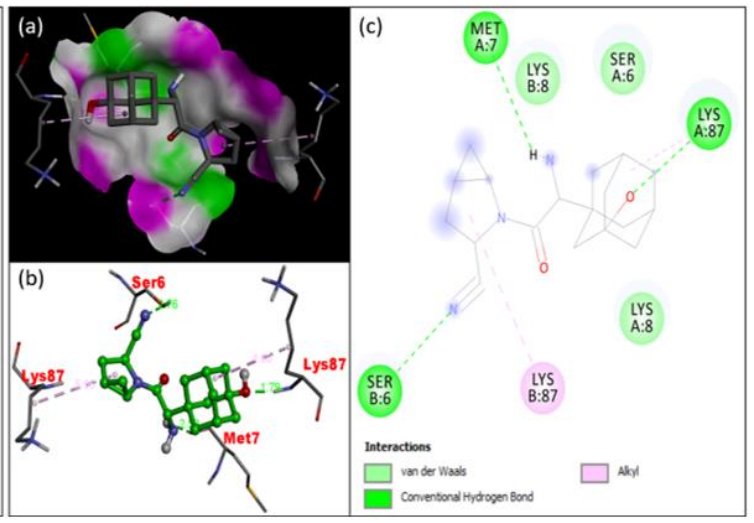

(b) Saxagliptin

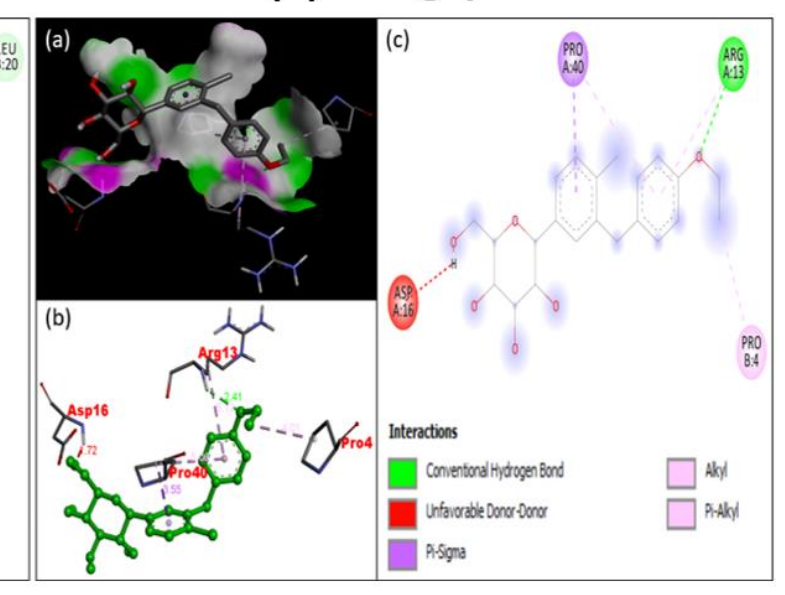

(d) Dapagliflozin

Figure 9. Molecular interaction of anti-diabetic drugs with active site residues of ZO-1 protein (PDB:3shu): (a) voglibose; (b) saxagliptin; (c) repaglinide; (d) dapagliflozin.

\section{Discussion}

Hyperglycaemia is a second leading world threat causing morbidities and mortalities in the world. People with diabetes have cognitive impairment due to metabolic disturbances and impaired brain insulin pathways; they activate inflammation and microglia activation, leading to a series of events causing an increase in ischemia risk factors, neurodegeneration, and cognitive decline. Diabetes has reported distorted endothelial functions associated with neurodegenerative disorders, progressive brain insult, and neuronal loss. Hyperglycaemia or 
diabetes type-2 (T2DM) is prone to develop cardiovascular and cerebrovascular complications. Brain ischemia or stroke and cognitive decline are the first-line morbidities associated with chronic hyperglycemia. Oxidative stress and neuronal loss are progressive pathologies in hyperglycemia. Brain ischemia and hyperglycemia are bidirectional pathology and progress synergistically. The current clinical research reports support using voglibose as monotherapy in type-2 diabetes and a healthy individual, which can reduce the incidence of a cardiovascular event by decreasing postprandial hyperglycemia [31,32]. Several studies have reported that treatment with voglibose also results in a significant decline in triglyceride levels and prevents atherosclerosis [33]. Based on accumulating evidence, which suggests that voglibose is an ideal cardioprotective agent, it may exert cerebral-protective effects. Saxagliptin, a dipeptidyl peptidase-4 inhibitor, is an oral hypoglycaemic agent that restrains the breakdown of the incretin hormones, GLP-1 and GDITP (glucose-dependent insulinotropic polypeptide) is widely prescribed and used as monotherapy and add-on therapy with other anti-hyperglycaemic agents for the better control over postprandial hyperglycemia. Repaglinide is a drug of class meglitinides that works in a similar manner to sulfonylurea and secretes the insulin from pancreatic beta cells for achieving the desired lowering sugar levels quickly. Physicians frequently prescribe it due to its quick glucose-lowering quality and strict postprandial glucose control. In previous research reports, Dapagliflozin, an SGLT2 inhibitor, has reduced apoptosis and inflammation (cultured ischemic tubular cells) in ischemic renal disease in mice[34].

Although the effects of SGLT-2 inhibitor over ischemia are unclear, several research reports have evident its efficacy in improving ischemia by improving cardiovascular risk factors[35].

From recent evidence and having proven strict glycemic control, we hypothesize to evaluate these four classes of drugs for their neuroprotective action through in-silico molecular simulation studies between stroke-associated proteins and selected anti-diabetic agents. We screened all the anti-diabetic agents with in-silico molecular docking and virtual screening with targeted proteins responsible for possible activation of impairment of various pathways leading to the diabetes-associated cerebrovascular complications, apoptosis, active inflammation, neurodegeneration, and neurodegeneration cognitive decline. We screened all four antidiabetic agents with bcl-2, caspase-3, caspase-8, caspase-9, IL-6, MAPK/ERK, TGF- $\beta$, TNF$\alpha, \mathrm{ZO}-1$ protein molecules obtained from the protein bank (RCSB.PDB). The interactions derived from in-silico molecular dynamic stimulation and virtual screening frameworks are often involved in the biological mechanism essential for receptor-ligand binding. More docking fitness allows the possible interaction with protein and its therapeutic action. We established ADD's structure-based virtual screening framework with proteins like caspase 3, 8, 9; IL-6, MAPK/ERK; PI3K, BCl-2, TNF- $\alpha$, TGF- $\beta$, ZO-1 proteins. It is well established that caspases 3 and 8 are the pre-apoptotic markers, and caspase 9 posts apoptosis marker [36]. In our study, docking of voglibose and saxagliptin with caspases showed a good docking score and binding energy, which predicts the expected neuroprotective effect through inhibition of apoptosis.

Extracellular signal-regulated kinase $1 / 2(E R K 1 / 2)$ is one of the best-characterized members of the mitogen-activated protein kinase (MAPK) family, which is associated with series of activities of the cell cycle, cell death, and survival. Previous research data on ERK1/2 indicates that it plays a vital role in inflammation and oxidative stress during brain injury and its reduced activity evident of ischemic damage[37,38]. Upon docking, voglibose and saxagliptin were found to have a good binding score and docking energy with MAPK/ERK 
and PI3K protein. Thus, docking results hypothesize the possible signaling pathway of voglibose and saxagliptin through ERK1/2.

Tumor necrosis factor-alpha ( $\mathrm{TNF} \alpha)$ is a cytokine synthesized by mostly macrophages and microglia during a cell injury event. The co-relation of Bcl-TNF-alpha in neurodegeneration and brain damage is intimately linked[39]. The dual activation of TNFalpha and Bcl family proteins induces neuronal cell apoptosis and mimics stroke pathology. The lower expression of both proteins is reciprocal to neuronal health and brain damage[40]. Our in-silico study found potent binding and docking scores of voglibose and saxagliptin with Bcl-2 and TNF- $\alpha$, which strongly predicts its clear involvement in anti-inflammatory activity.

Bloodstream and cerebrospinal fluid levels rise immediately following a stroke, and observational studies specify that stroke lesion size correlates with brain injury-related proteins like TGF- $\beta$, ZO-1 levels[41]. Voglibose and saxagliptin showed good docking energy and docking score with TGF- $\beta$, ZO-1 proteins, promising therapeutic potential against stroke and brain injury.

The molecular docking study found that voglibose, saxagliptin, repaglinide and Dapagliflozin have a greater negative binding score with Bcl-2 and IL-6 protein. With evidence to these docking reports, we hypothesize their role as active anti-inflammatory agents by inducing beneficial anti-inflammatory responses over hyperglycemic conditions. By Molecular docking with caspase cascade and anti-diabetic agents, we got higher docking affinity and better docking score with caspase-3, caspase-8, and caspase-9. The results of docking binding affinity of anti-diabetic agents and caspase cascade proteins show its possible anti-apoptotic role over hyperglycaemic conditions. Molecular docking with MAPK/ERK protein and antidiabetic agents resulted in a great binding score, docking energy, and hydrogen binding, giving us an idea of its possible role in the MAPK/ERK pathway, polyol pathway, PKC pathway, and oxidative stress. The results of docking with stroke-associated proteins (TNF- $\alpha$, TGF- $\beta$, ZO-1) were also promising with all anti-diabetic agents. The results show a very good affinity and docking score with stroke-associated proteins, which gives us an evident hypothesis of its involvement in diabetes-associated ischemia. The molecular docking simulation study results demonstrated the evident pilot role of their possible potent therapeutic action of all four antidiabetic agents: anti-diabetic, anti-inflammatory, anti-apoptosis, and anti-stroke activity[42,43].

\section{Conclusions}

In-silico molecular simulation study of anti-diabetic agents with stroke and neurodegeneration associated proteins gave a strong hypothesis to the neuroprotective actions of the anti-diabetic drugs. Moreover, higher binding energy with all biomarker proteins directs us of exerting strong therapeutic action of the selected class of anti-diabetic agents, i.e., voglibose, saxagliptin, repaglinide, and Dapagliflozin with BCl-2, Caspase-3, Caspase-8, Caspase-9, IL6, MAPK/ERK, PI3K, TGF- $\beta$, TNF- $\alpha$ and ZO-1. That indicates its potential role against inflammation, neurodegeneration, and apoptotic cascade in hyperglycemic brain ischemic conditions through various diabetic pathogenic pathways.

\section{Funding}

This research received no external funding. 


\section{Acknowledgments}

Author (Vishal K Chavda) would like to acknowledge CSIR (Council for Scientific and Industrial Research) for providing CSIR-SRF (Senior Research Fellowship -Ack/no. 111115/2K18/1; File Number 09/1048(0011)/2019-EMR-1) as partial funding to support original research work.

\section{Conflicts of Interest}

The authors declare no conflict of interest.

\section{References}

1. Yazar, M.; Özbek, P. In Silico Tools and Approaches for the Prediction of Functional and Structural Effects of Single-Nucleotide Polymorphisms on Proteins: An Expert Review. Omi. A J. Integr. Biol.2021, 25, 2337,https://doi.org/10.1089/omi.2020.0141.

2. Agarwal, N.; Chetry, V.; Poluri, K.M. Elucidating Protein-Ligand Interactions Using High Throughput Biophysical Techniques. In Innovations and Implementations of Computer Aided Drug Discovery Strategies in Rational Drug Design; Springer Singapore, 2021; pp 205-244,https://doi.org/10.1007/978-981-15-89362_9.

3. Sulimov, V.B.; Kutov, D.C.; Taschilova, A.S.; Ilin, I.S.; Tyrtyshnikov, E.E.; Sulimov, A.V. Docking Paradigm in Drug Design. Curr. Top. Med. Chem.2020, 21, 507546,https://doi.org/10.2174/1568026620666201207095626.

4. Naqvi, A.A.T.; Mohammad, T.; Hasan, G.M.; Hassan, M.I. Advancements in Docking and Molecular Dynamics Simulations Towards Ligand-Receptor Interactions and Structure-Function Relationships. Curr. Top. Med. Chem.2019, 18, 1755-1768,https://doi.org/10.2174/1568026618666181025114157.

5. Air, E.L.; Kissela, B.M. Diabetes, the Metabolic Syndrome, and Ischemic Stroke: Epidemiology and Possible Mechanisms. Diabetes Care2007, 30, 3131-3140,https://doi.org/10.2337/dc06-1537.

6. Lyu, F.; Wu, D.; Wei, C.; Wu, A. Vascular Cognitive Impairment and Dementia in Type 2 Diabetes Mellitus: An Overview. Life Sci.2020, 254, 117771,https://doi.org/10.1016/j.lfs.2020.117771.

7. Chen, R.; Ovbiagele, B.; Feng, W. Diabetes and Stroke: Epidemiology, Pathophysiology, Pharmaceuticals and Outcomes. Am. J. Med. Sci.2016, 351, 380-386,https://doi.org/10.1016/j.amjms.2016.01.011.

8. Li, P.; Quan, W.; Lu, D.; Wang, Y.; Zhang, H.H.; Liu, S.; Jiang, R.C.; Zhou, Y.Y. Association between Metabolic Syndrome and Cognitive Impairment after Acute Ischemic Stroke: A Cross-Sectional Study in a Chinese Population. PLoS One2016, 11,e0167327, https://doi.org/10.1371/journal.pone.0167327.

9. van Sloten, T.T.; Sedaghat, S.; Carnethon, M.R.; Launer, L.J.; Stehouwer, C.D.A. Cerebral Microvascular Complications of Type 2 Diabetes: Stroke, Cognitive Dysfunction, and Depression. Lancet Diabetes Endocrinol.2020, 8, 325-336,https://doi.org/10.1016/S2213-8587(19)30405-X.

10. Murphy, J. Pharmacological Treatment of Acute Ischemic Stroke. Crit. Care Nurs. Q.2003, 26, 276-282, https://doi.org/10.1097/00002727-200310000-00003.

11. Ho, N.; Sommers, M. S.; Lucki, I. Effects of Diabetes on Hippocampal Neurogenesis: Links to Cognition and Depression. Neurosci. Biobehav. Rev.2013, 37, 1346-1362,https://doi.org/10.1016/j.neubiorev.2013.03.010.

12. Westphal, L.P.; Held, U.; Engelter, S.; Wegener, S. Association of Prestroke Metformin Use, Stroke Severity, and Thrombolysis $\quad$ Outcome. $\quad$ Neurology2021, $\quad 96, \quad 362-$ 373,https://doi.org/10.1212/WNL.0000000000011563.

13. Gohil, C.J.; Noolvi, M.N. Design and in Silico Study of the Novel Small Molecular Mdm2 Inhibitors. Biointerface Res. Appl. Chem.2021, 11, 8052-8064,https://doi.org/10.33263/BRIAC111.80528064.

14. Trott, O.; Olson, A.J. AutoDock Vina: Improving the Speed and Accuracy of Docking with a New Scoring Function, Efficient Optimization, and Multithreading. J. Comput. Chem.2009, 31, 455461,https://doi.org/10.1002/jcc.21334.

15. Wang, Z.; He, N.; Guo, Z.; Niu, C.; Song, T.; Guo, Y.; Cao, K.; Wang, A.; Zhu, J.; Zhang, X.; Zhang, Z. Proteolysis Targeting Chimeras for the Selective Degradation of Mcl-1/Bcl-2 Derived from Nonselective Target Binding Ligands. J. Med. Chem.2019, 62, 8152-8163, https://doi.org/10.1021/acs.jmedchem.9b00919.

16. Singh, S.K.; Upadhyay, A.K.; Reddy, M.S. Screening of Potent Drug Inhibitors against SARS-CoV-2 RNA Polymerase: An in Silico Approach. 3 Biotech2021, 11, 93,https://doi.org/10.1007/s13205-020-02610-w.

https://biointerfaceresearch.com/ 
17. Patel, V.; Shah, J.; Gupta, A. Design and In-Silico Study of Bioimaging Fluorescence Graphene Quantum Dot-Bovine Serum Albumin Complex Synthesized by Diimide-Activated Amidation. Comput. Biol. Chem.2021, 93, 107543,https://doi.org/https://doi.org/10.1016/j.compbiolchem.2021.107543.

18. Worawalai, W.; Sompornpisut, P.; Wacharasindhu, S.; Phuwapraisirisan, P. Voglibose-Inspired Synthesis of New Potent $\alpha$-Glucosidase Inhibitors N-1,3-Dihydroxypropylaminocyclitols. Carbohydr. Res. 2016, 429, 155-162,https://doi.org/10.1016/j.carres.2016.04.014.

19. Kalhotra, P.; Chittepu, V.C.S.R.; Osorio-Revilla, G.; Gallardo-Velázquez, T. Structure-Activity Relationship and Molecular Docking of Natural Product Library Reveal Chrysin as a Novel Dipeptidyl Peptidase-4 (DPP4) Inhibitor: An Integrated in Silico and in Vitro Study. Molecules2018, 23,1368, https://doi.org/10.3390/molecules23061368.

20. Kulkarni, S.; Gupta, P.; Pallavi, A. Investigation of Enzymes Binding to "Voglibose- an Anti-diabetic Drug" and the Choice of Enzyme to Be Used for Biosensing. Br. J. Pharm. Res.2016, 14,https://doi.org/10.9734/bjpr/2016/30369.

21. Boatright, K.M.; Salvesen, G.S. Mechanisms of Caspase Activation. Curr. Opin. Cell Biol.2003, 15, 725731. https://doi.org/10.1016/j.ceb.2003.10.009.

22. Yao, L.; Swartz, P.; Hamilton, P.T.; Clark, A.C. Remodeling Hydrogen Bond Interactions Results in Relaxed Specificity of Caspase-3. Biosci. Rep.2021, 41, 20203495,https://doi.org/10.1042/BSR20203495.

23. Park, H.H. Molecular Basis of Dimerization of Initiator Caspase Was Revealed by Crystal Structure of Caspase-8 pro-Domain. Cell Death Differ.2019, 26, 1213-1220,https://doi.org/10.1038/s41418-018-0200-x.

24. Renatus, M.; Stennicke, H.R.; Scott, F.L.; Liddington, R.C.; Salvesen, G.S. Dimer Formation Drives the Activation of the Cell Death Protease Caspase 9. Proc. Natl. Acad. Sci. U. S. A.2001, 98, 1425014255,https://doi.org/10.1073/pnas.231465798.

25. Akbari, M.; Hassan-Zadeh, V. IL-6 Signalling Pathways and the Development of Type 2 Diabetes. Inflammopharmacology2018, 26, 685-698,https://doi.org/10.1007/s10787-018-0458-0.

26. Veverka, V.; Baker, T.; Redpath, N.T.; Carrington, B.; Muskett, F.W.; Taylor, R.J.; Lawson, A.D.G.; Henry, A.J.; Carr, M.D. Conservation of Functional Sites on Interleukin-6 and Implications for Evolution of Signaling Complex Assembly and Therapeutic Intervention. J. Biol. Chem.2012, 287, 4004340050,https://doi.org/10.1074/jbc.M112.405597.

27. Bhargavi, M.; Vhora, N.; Lanka, G.; Somadi, G.; Kanth, S.S.; Jain, A.; Potlapally, S. R. Homology Modelling and Virtual Screening to Explore Potent Inhibitors for MAP2K3 Protein. Struct. Chem.2021, 32, 10391051,https://doi.org/10.1007/s11224-020-01667-w.

28. Choodamani, B.; Cano Hernandez, K.G.; Kumar, S.; Tony, A.M.; Schiaffino Bustamante, A.Y.; Aguilera, R.J.; Schols, D.; Gopi Mohan, C.; Karki, S.S. Synthesis, Molecular Docking and Preliminary Antileukemic Activity of 4-Methoxybenzyl Derivatives Bearing Imidazo[2,1-b][1,3,4]Thiadiazole. Chem. Biodivers.2021, 18, e2000800,https://doi.org/10.1002/cbdv.202000800.

29. Khajouei, A.; Hosseini, E.; Abdizadeh, T.; Kian, M.; Ghasemi, S. Beneficial Effects of Minocycline on the Ovary of Polycystic Ovary Syndrome Mouse Model: Molecular Docking Analysis and Evaluation of TNF$\alpha$, TNFR2, TLR-4 Gene Expression. J. Reprod. Immunol.2021, 144, 103289,https://doi.org/10.1016/j.jri.2021.103289.

30. Singh, S.P.; Nongalleima, K.; Singh, N.I.; Chanu, W.K.; Singh, T.R.; Singh, C.B. Computational Investigation of Zerumbone as an Inhibitor of TNF-Alpha Using Molecular Dynamics and Molecular Docking Methods. Lett. Drug Des. Discov.2020, $18, \quad 258-$ 268,https://doi.org/10.2174/1570180817999201110112221.

31. Gerstein, H.C.; Coleman, R.L.; Scott, C.A.B.; Xu, S.; Tuomilehto, J.; Rydén, L.; Holman, R.R. Impact of Acarbose on Incident Diabetes and Regression to Normoglycemia in People with Coronary Heart Disease and Impaired Glucose Tolerance: Insights from the Ace Trial. Diabetes Care2020, 43, 22422247,https://doi.org/10.2337/dc19-2046.

32. Vashi, R.; Chavda, V.; Patel, S.S. Cerebrovascular Complications of Diabetes: SGLT-2 Inhibitors as a Promising Future Therapeutics. Curr. Drug Targets2020, 22, 16291636,https://doi.org/10.2174/1389450121666201020163454.

33. Creager, M.A.; Lüscher, T.F.; Cosentino, F.; Beckman, J.A. Diabetes and Vascular Disease. Pathophysiology, Clinical Consequences, and Medical Therapy: Part I. Circulation2003, 108, 1527-1532, https://doi.org/10.1161/01.CIR.0000091257.27563.32.

34. Chang, Y.K.; Choi, H.; Jeong, J.Y.; Na, K.R.; Lee, K.W.; Lim, B.J.; Choi, D.E. Dapagliflozin, SGLT2 Inhibitor, Attenuates Renal Ischemia-Reperfusion Injury. PLoS One2016, 11, 
e01588010,https://doi.org/10.1371/journal.pone.0158810.

35. Sa-nguanmoo, P.; Tanajak, P.; Kerdphoo, S.; Jaiwongkam, T.; Pratchayasakul, W.; Chattipakorn, N.; Chattipakorn, S. C. SGLT2-Inhibitor and DPP-4 Inhibitor Improve Brain Function via Attenuating Mitochondrial Dysfunction, Insulin Resistance, Inflammation, and Apoptosis in HFD-Induced Obese Rats. Toxicol. Appl. Pharmacol.2017, 333, 43-50,https://doi.org/10.1016/j.taap.2017.08.005.

36. Elmore, S. Apoptosis: A Review of Programmed Cell Death. Toxicol. Pathol.2007, 35, 495-516, https://doi.org/10.1080/01926230701320337.

37. Sawe, N.; Steinberg, G.; Zhao, H. Dual Roles of the MAPK/ERK1/2 Cell Signaling Pathway after Stroke. $J$. Neurosci. Res.2008, 86, 1659-1669,https://doi.org/10.1002/jnr.21604.

38. Boulton, T.G.; Nye, S.H.; Robbins, D.J.; Ip, N.Y.; Radzlejewska, E.; Morgenbesser, S.D.; DePinho, R. A.; Panayotatos, N.; Cobb, M.H.; Yancopoulos, G.D. ERKs: A Family of Protein-Serine/Threonine Kinases That Are Activated and Tyrosine Phosphorylated in Response to Insulin and NGF. Cell1991, 65, 663675,https://doi.org/10.1016/0092-8674(91)90098-J.

39. Ibrahim, R.R.; El-Esawy, R.O; El-Sakaa, M.H. Troxerutin Downregulates C/EBP- $\beta$ Gene Expression via Modulating the IFN $\gamma$-ERK1/2 Signaling Pathway to Ameliorate Rotenone-Induced Retinal Neurodegeneration. J. Biochem. Mol. Toxicol.2020, 34, e22482,https://doi.org/10.1002/jbt.22482.

40. Borlongan, M.C.; Kingsbury, C.; Salazar, F.E.; Toledo, A.R.L.; Monroy, G.R.; Sadanandan, N.; Cozene, B.; Gonzales-Portillo, B.; Saft, M.; Wang, Z.-J.; Moscatello, A.; Lee, J.Y. IL-2/IL-2R Antibody Complex Enhances Treg-Induced Neuroprotection by Dampening TNF- $\alpha$ Inflammation in an In Vitro Stroke Model. NeuroMolecular Med.2021, https://doi.org/10.1007/s12017-021-08656-0.

41. Baeten, K.M.; Akassoglou, K. Extracellular Matrix and Matrix Receptors in Blood-Brain Barrier Formation and Stroke. Dev. Neurobiol.2011, 71, 1018-1039,https://doi.org/10.1002/dneu.20954.

42. Prabhakaran, D.; Anand, S.; Watkins, D.; Gaziano, T.; Wu, Y.; Mbanya, J.C.; Nugent, R.; Ajay, V.S.; Afshin, A.; Adler, A.; Ali, M.K.; Bateman, E.; Bettger, J.; Bonow, R.O.; Brouwer, E.; Bukhman, G.; Bull, F.; Burney, P.; Capewell, S.; Chan, J.; Chandrasekar, E.K.; Chen, J.; Criqui, M.H.; Dirks, J.; Dugani, S.B.; Engelgau, M.; El Nahas, M.; Fall, C.H.; Feigin, V.; Fowkes, F.G.R.; Glassman, A.; Goenka, S.; Gupta, R.; Hasan, B.; Hersch, F.; Hu, F.; Huffman, M.D.; Jabbour, S.; Jarvis, D.; Jeemon, P.; Joshi, R.; Kamano, J.H.; Kengne, A.P.; Kudesia, P.; Kumar, R.K.; Kumaran, K.; Lambert, E.V.; Lee, E.S.; Li, C.; Luo, R.; Magee, M.; Malik, V.S.; Marin-Neto, J.A.; Marks, G.; Mayosi, B.; McGuire, H.; Micha, R.; Miranda, J.J.; Montoya, P.A.; Moran, A.E.; Mozaffarian, D.; Naicker, S.; Naidoo, N.G.; Narayan, K.V.; Nikolic, I.; O’Donnell, M.; Onen, C.; Osmond, C.; Patel, A.; Perez-Padilla, R.; Poulter, N.; Pratt, M.; Rabkin, M.; Rajan, V.; Rassi, A.; Rassi, A.; Rawal, I.; Remuzzi, G.; Riella, M.; Roth, G. A.; Roy, A.; Rubinstein, A.; Sakuma, Y.; Sampson, U.K.; Siegel, K.R.; Sliwa, K.; Suhrcke, M.; Tandon, N.; Thomas, B.; Vaca, C.; Vedanthan, R.; Verguet, S.; Webb, M.; Weber, M.B.; Whitsel, L.; Wong, G.; Yan, L.L.; Yancy, C.W.; Zhang, P.; Zhao, D.; Zhu, Y. Cardiovascular, Respiratory, and Related Disorders: Key Messages from Disease Control Priorities, 3rd Edition. Lancet 2018, 391, 1224-1236,https://doi.org/10.1016/S0140-6736(17)32471-6.

43. Bharatam, P.; Patel, D.; Adane, L.; Mittal, A.; Sundriyal, S. Modeling and Informatics in Designing AntiDiabetic Agents. Curr. Pharm. Des.2007, 13, 3518-3530,https://doi.org/10.2174/138161207782794239. 